\title{
Análisis de la brecha de aprendizaje entre indígenas y no indígenas en la enseñanza primaria en México
}

\section{Analysis of the Learning Gap between Indigenous and Non-Indigenous Elementary Students in Mexico}

\author{
Emilio Blanco (1) eblanco@colmex.mx \\ (1) El Colegio de México \\ (Recibido: 19 de abril de 2017; Aceptado para su publicación: 8 de junio de 2017)
}

Cómo citar: Blanco, E. (2019). Análisis de la brecha de aprendizaje entre indígenas y no indígenas en la enseñanza primaria en México. Revista Electrónica de Investigación Educativa, 21, e16, 1-15. doi.10.24320/redie.2019.21.e16.1941

\section{Resumen}

El objetivo de este artículo es estimar el peso relativo que -sobre la desventaja de aprendizajes de los alumnos indígenas de enseñanza primaria en México- tienen los factores socioeconómicos, escolares y culturales. Para esto se especifican modelos jerárquico-lineales y Oaxaca-Blinder, para analizar y descomponer la brecha de aprendizajes entre alumnos indígenas y no indígenas, sobre los microdatos del Plan Nacional para la Evaluación de los Aprendizajes (PLANEA) 2015. Los principales resultados son: 1) además de las diferencias entre individuos, una parte importante de las diferencias se explica por el porcentaje de alumnos indígenas en las escuelas (efecto composicional); 2) estas diferencias se reducen significativamente al controlar por factores socioeconómicos y escolares (especialmente, por los primeros); 3) si bien los factores socioeconómicos dan cuenta de la mayor parte de la brecha, la trayectoria escolar de los alumnos y las características de las escuelas a las que asisten, adquieren mayor peso para explicar las diferencias en matemáticas, y la desventaja específica de los alumnos que aprendieron primero una lengua indígena.

Palabras clave: Brecha de aprendizaje, población indígena, enseñanza primaria.

\section{Abstract}

The objective of this article is to estimate the relative weight of socioeconomic, academic and cultural factors in the learning disadvantages of indigenous elementary students in Mexico. To this end, hierarchical linear and Oaxaca-Blinder models were specified to analyze and decompose the learning gap between indigenous and non-indigenous students, on the basis of microdata from the National Plan for Learning Assessment (PLANEA) 2015. The main results are 1) beyond individual differences, many of these differences are explained by the percentage of indigenous students in schools (the compositional effect); 2) these differences are greatly reduced when socioeconomic and academic factors are controlled for (especially the former); and 3) although socioeconomic factors account for most of the gap, students' academic trajectories and the characteristics of the schools they attend take on greater relevance in 
explaining differences in mathematics and the specific disadvantage experienced by students who learned an indigenous language first.

Keywords: Learning gap, indigenous peoples, primary education.

\section{Introducción}

La población indígena en México vive en condiciones sustancialmente inferiores a las del resto de los habitantes. Independientemente del criterio de identificación que se adopte ${ }^{1}$, los indígenas presentan cifras de pobreza extrema y carencias sociales entre dos y cuatro veces más elevadas que las de la población no indígena, al punto que casi la totalidad de los indígenas son pobres o vulnerables (Consejo Nacional de Evaluación de la Política de Desarrollo Social [CONEVAL] 2014). La desventaja educativa de los indígenas es una dimensión central de esta situación de marginación; las brechas entre indígenas y el resto de la población sólo se han equiparado en el acceso al nivel primario (Instituto Nacional para la Evaluación de la Educación [INEE] 2016a). En el resto de los indicadores (acceso a niveles secundario, medio superior y terciario; rezago, reprobación, abandono) el rezago persiste (INEE, 2016a).

Si se consideran los resultados educativos, el Plan Nacional para la Evaluación de los Aprendizajes (Planea, 2015) muestra que mientras a nivel nacional el porcentaje de alumnos ubicados en niveles insuficientes de aprendizaje alcanza el 49.5 y 60.5\% (Lengua y Matemáticas, respectivamente), entre los alumnos que asisten a escuelas indígenas estas cifras son, respectivamente, de 80 y 83\% (INEE, 2016a). Explicar estas diferencias en los aprendizajes es el objetivo de este artículo; específicamente, busco descomponer estas diferencias para determinar qué parte puede ser explicada por distintos grupos de factores, a fin de contribuir a la discusión sobre los mecanismos involucrados en la producción de la desigualdad educativa.

A grandes rasgos, es posible identificar cinco explicaciones, no excluyentes, sobre la desventaja de aprendizajes de los indígenas.

La primera explicación se basa en la robusta relación entre factores socioeconómicos y aprendizajes (Cervini 2012; Fernández 2007), que convierte a los primeros en candidatos obvios. Bajo este escenario, controlar las brechas iniciales por una batería de factores socioeconómicos debería reducir sustancialmente a aquéllas. La principal hipótesis general que sostiene este artículo es, efectivamente, que los factores socioeconómicos logran explicar la mayor parte de las brechas de aprendizaje; en otras palabras, estas brechas responden más a las diferencias en los recursos materiales y educativos de los grupos analizados, que a procesos de discriminación o irrelevancia cultural de los modelos escolares.

En segundo lugar, destaca la desigualdad en las características de las escuelas a las que asisten alumnos indígenas y no indígenas. Esta explicación enfatiza las carencias en infraestructura (INEE, 2016b), la organización incompleta o multigrado de muchas de estas escuelas (INEE, 2016a), o el menor nivel de formación de los maestros (INEE, 2015; Santibáñez 2016; Schmelkes, 2013). Si bien esta explicación puede forzarse dentro de un esquema de "discriminación sistémica", considero que es más adecuado considerarlo una expresión de desventaja de recursos institucionalizada.

La tercera explicación es la falta de pertinencia y relevancia cultural del modelo educativo implementado para los alumnos indígenas. A pesar de que en las últimas décadas se han abandonado las aproximaciones "asimiladoras" en favor de un modelo intercultural bilingüe (Bertely 2002), las dificultades en su implementación práctica en las escuelas indígenas (Bastiani et al., 2012; Tinajero y Englander, 2011), así como el hecho de que muchos alumnos indígenas asisten a escuelas de tipo general, desembocan -según esta teoría- en la exposición a modelos poco pertinentes, que terminan por alienar a los alumnos de los procesos de aprendizaje.

En cuarto lugar, se ha enfatizado la discriminación que sufren los alumnos indígenas dentro de las escuelas, especialmente en las escuelas no indígenas. Numerosos autores han documentado la

\footnotetext{
${ }^{1}$ Según el Instituto Nacional de Estadística y Geografía (INEGI) los hablantes de alguna lengua indígena ascendían en 2015 a 6.5\%, y quienes se autoidentifican como indígenas alcanzaban el 21.5\% (INEGI, 2015).
} 
invisibilización y/o inferiorización de que son objeto por parte de maestros y compañeros, lo que genera sentimientos de vergüenza, malestar escolar, y actitudes de retraimiento (Barriga 2008; Mijangos-Noh 2009; Raesfeld 2009; Schmelkes 2013). Si esta discriminación se traduce efectivamente en desigualdades de aprendizajes, debería observarse una brecha significativa entre indígenas y no indígenas, a pesar de controlar por factores socioeconómicos y escolares; otro indicio de prácticas de discriminación podría ser una brecha mayor en las escuelas generales que en las escuelas indígenas.

La quinta explicación es, simplemente, que los alumnos indígenas están siendo evaluados en (y, en el caso de las pruebas de lenguaje, sobre) un idioma del cual no tienen un dominio comparable al de los hispanohablantes nativos (Treviño, 2013). Si bien parece de sentido común, la escasa investigación empírica disponible muestra que éste no siempre podría ser el caso. ${ }^{2}$ En el contexto de esta investigación, un elemento de prueba favorable a esta teoría debería ser una mayor brecha en lenguaje que en matemáticas, tanto antes como después de introducir controles estadísticos.

Dejando de lado la última hipótesis, las dos primeras podrían denominarse "estructurales", mientras que las dos siguientes podrían denominarse "culturales". Si bien la hipótesis básica de este artículo es que las primeras obtienen mayor apoyo empírico que las segundas, deben tenerse en cuenta dos aspectos:

1) No se cuenta con variables para evaluar directamente las hipótesis "culturales", por lo que es necesario recurrir a supuestos para fundamentar su observación indirecta. Podría suponerse, por ejemplo, que la totalidad de las brechas remanentes luego de controlar por las variables "estructurales" podría atribuirse a mecanismos "culturales"; sin embargo, la posibilidad de variables estructurales no observadas que inciden en los aprendizajes sugiere tomar a la brecha remanente como una estimación "máxima" de los efectos culturales.

2) La separación entre efectos estructurales y efectos culturales no necesariamente puede hacerse de forma nítida, dado que puede haber interacciones entre ellos (por ejemplo: los alumnos indígenas podrían sufrir mayor discriminación cuanto menor es su posición socioeconómica).

Las investigaciones para Latinoamérica muestran variaciones considerables en las brechas y la capacidad explicativa de distintos factores, dependiendo de los países estudiados, los datos utilizados, y las áreas de aprendizaje consideradas. Lo anterior justifica el esfuerzo de profundizar en esta línea de investigación.

En una revisión de estudios, McEwan y Trowbridge (2007) reportan diferencias de 1.1 a 0.2 desvíos estándar, según el país y el área evaluados. Para las primarias de Guatemala, específicamente, los autores mostraron disminuciones significativas en las brechas, una vez controladas las variables socioeconómicas y los efectos escolares. Las características de las escuelas, en particular, explicarían alrededor de dos tercios de las brechas; finalmente, entre 24 y $45 \%$ de las mismas permanecen sin explicar. Por otro lado, un estudio para el nivel primario de Perú arrojó brechas de aprendizaje de entre 0.5 y 1.2 desvíos estándar, que resultaron casi completamente explicadas por factores socioeconómicos (Sakellariou, 2008). Reducciones similares se han reportado en Bolivia y Chile, para diferencias iniciales de entre $0.3 \mathrm{y}$ 0.5 desvíos estándar (McEwan, 2004). En un estudio sobre Guatemala, Perú y México, Hernández-Zavala, Patrinos, Sakellariou y Shapiro (2006) reportaron, para este último caso, brechas de 0.7 desvíos estándar en español y matemáticas, que se reducen en $70 \%$ al controlar por variables individuales y escolares (sobre todo, por las primeras).

El único estudio en México que recure a una metodología de descomposición de brechas (específicamente, para el estado de Chiapas) fue publicado por Santibáñez (2016), quien halló que las características socioeconómicas de los estudiantes explican 60-70\% de las diferencias entre indígenas y no indígenas.

\footnotetext{
${ }^{2}$ Para una muestra de alumnos mayas, Backhoff, Contreras, Vázquez y Sánchez (2015) reportaron la ausencia de correlación entre la familiaridad con la lengua maya y el desempeño en una prueba de español.
} 


\section{Método}

Para el análisis de los datos se recurrió a modelos multinivel (Raudenbush y Bryk, 2002) y a descomposiciones Oaxaca-Blinder (O-B) (Blinder, 1973; Oaxaca, 1973). En ambos casos, se parte de una brecha inicial (o bruta) de aprendizajes entre indígenas y no indígenas, para proceder a explicarla estadísticamente mediante la introducción de variables e identificar qué proporciones de la explicación corresponden a distintos grupos de factores.

Los datos provienen de la prueba Planea 2015 para sexto año de primaria. Únicamente se incluyeron los casos con información completa, por lo que los tamaños finales de las muestras fueron de 80,080 individuos y 2,735 escuelas para los modelos multinivel ${ }^{3}$, y de 81,389 individuos y 2,952 escuelas para las descomposiciones O-B. ${ }^{4}$

Si bien no se dispone de variables para observar fenómenos como la discriminación o la irrelevancia cultural del modelo educativo, en principio sería posible estimar de manera indirecta los efectos de eventuales procesos de este tipo. Habitualmente, la proporción de la brecha no explicada por los modelos O-B se toma como un indicador general de discriminación (McEwan, 2004), debido a que apunta a diferencias en los rendimientos de los recursos o experiencias de ambos grupos (por oposición a diferencias explicadas por diferencias de recursos y experiencias). Sin embargo, dado que potencialmente existen variables relevantes no incluidas en el modelo, aunado a la ya mencionada desventaja propia de resolver pruebas en español, así como a la posibilidad de que las variables observadas únicamente se pueden considerar proxies de los factores relevantes (por ejemplo, el nivel educativo de los padres no indica la calidad de la educación adquirida), parece más cauto interpretar esta proporción como un "techo máximo posible" para los eventuales efectos de discriminación/irrelevancia cultural.

Más allá de esto, y por razones que se exponen en las conclusiones, en este trabajo se sostiene que para las desigualdades de aprendizaje incluso esta interpretación es aventurada. La complejidad de los factores involucrados no permite distinguir netamente entre "efectos de recursos" y "efectos de rendimientos". No obstante, el método O-B permite separar grupos de factores con cierta nitidez, por lo que puede evaluarse la plausibilidad de algunos mecanismos explicativos.

Las variables dependientes en los modelos son los puntajes estandarizados de las pruebas de lenguaje/comunicación y matemáticas. Las variables independientes se dividen en variables de nivel individual y variables de nivel escolar. ${ }^{5}$

A nivel individual se utilizaron las siguientes variables:

1) Alumno aprendió primero una lengua indígena: 0=No; $1=$ Sí.

2) Alumno se considera indígena: $0=\mathrm{No} ; 1=\mathrm{Sí}^{6}$

\footnotetext{
${ }^{3}$ Se excluyeron los cursos comunitarios del Consejo Nacional de Fomento Educativo (Conafe) dado que no cuentan con cuestionario de directores, lo que implicaba perder información relevante sobre las escuelas.

${ }^{4}$ La diferencia en los tamaños de muestra de individuos entre los modelos jerárquicos y las descomposiciones O-B responde a que, en el primer caso, se realizó un proceso de estimación con imputación de valores perdidos (mediante el módulo mi de Stata) para la variable "Nivel educativo del hogar", los cuales ascendían a 15.1\% de la muestra. Cada modelo jerárquico se estimó con un total de cinco imputaciones, criterio con el cual diferentes ejercicios arrojaron diferencias en los coeficientes para el tercer decimal, sin que se observen diferencias en los niveles de significación. Este procedimiento no puede realizarse con los modelos O-B porque el software no lo habilita, por lo que en este caso se eliminaron los casos con datos faltantes.

${ }^{5}$ Algunas de las variables de nivel escolar se observan a nivel individual, y se introducen de esa forma y como agregados a nivel escolar. Es el caso del índice de bienes en el hogar, el índice de clima de aula, y las dos variables que identifican a los alumnos indígenas.

${ }^{6}$ La posibilidad de distinguir entre quienes hablan una lengua indígena y quienes se identifican como tales abre perspectivas interesantes. En primer lugar, porque "desencializa" la condición indígena y abre un espectro de posibilidades de categorización, y en segundo lugar porque separa los aspectos específicamente lingüísticos de los
} 
3) Alumna es Mujer: 0=No; $1=$ Sí.

4) Bienes en el hogar del alumno: índice factorial tetracórico de bienes en el hogar (luz eléctrica, gas, teléfono, televisión, televisión de paga, Internet, computadora, lavadora, refrigerador, microondas, DVD o Blu-Ray, automóvil o camioneta).

5) Máximo nivel educativo de los padres del alumno: $0=$ Hasta primaria; $1=$ Secundaria 0 bachillerato; 2=Universidad o más.

6) Situación laboral del alumno: 0=No trabaja; 1=Trabaja en el hogar; 2=Trabaja fuera del hogar.

7) Asistencia a preescolar: $0=$ No asistió; $1=1$ año; $2=2$ años; $3=3$ años.

8) Reprobó algún grado: 0=No; $1=$ =Sí.

9) Expectativas educativas: 0=Cursar hasta bachillerato; 1=Cursar universidad; $2=$ Cursar posgrado.

10) Clima de aula percibido por el alumno: índice factorial policórico a partir de las siguientes preguntas: "Mi maestro... i) Toma en cuenta mis opiniones durante las clases"; ii) Me da confianza para preguntar mis dudas en clase"; iii) Organiza actividades en las que podemos dar nuestra opinión y escuchar las de los otros"; iv) Toma en cuenta nuestra opinión sobre las reglas en el salón de clases".

A nivel escolar las variables fueron:

11) Modalidad escolar: $0=$ =General pública; 1 = Indígena; $2=$ =Privada.

12) Grado evaluado es multigrado: $0=$ No; $1=$ Sí.

13) Director de la escuela tiene grupo a cargo: 0=No; $1=$ Sí.

14) Carencia de materiales educativos en la escuela: índice factorial policórico a partir de la existencia y suficiencia de mobiliario y materiales (mobiliario para sentarse y escribir, libros de lectura y consulta, computadoras o tablets, televisores, reproductores de video).

15) Porcentaje de alumnos que hablan una lengua indígena (promedio de \#1).

16) Porcentaje de alumnos que se identifican como indígenas (promedio de \#2).

17) Promedio escolar de bienes en el hogar de los alumnos (promedio de \#4).

18) Promedio escolar de clima de aula (promedio de \#10).

19) Altas expectativas educativas de los maestros hacia los alumnos (variable dicotómica: $1=$ Todos los maestros entrevistados esperan que sus alumnos accedan al nivel terciario; $0=$ Todos los demás casos).

culturales. Aunque la autoadscripción es un criterio controvertido porque, entre otras cosas, podría incluir un "sesgo" a favor de sujetos con mayor capital cultural (Villareal, 2014), cabe preguntarse si este "sesgo" no es en realidad parte del fenómeno que se pretende observar: la autoadscripción indígena como el resultado de un conjunto de dinámicas culturales relevantes, cuyas consecuencias para los procesos escolares, más que ser "controladas" deben ser tenidas en cuenta como tales. 


\section{Resultados}

\subsection{Descriptivos}

En la muestra utilizada para los análisis, $13.1 \%$ de los niños se autoidentifican como indígenas y 2.4\% declaran haber aprendido primero una lengua indígena. ${ }^{7}$ En la tabla I se muestra la distribución de las variables relevantes para cada grupo.

Si bien la probabilidad de encontrarse en una escuela indígena es mayor para ambos grupos que para los no indígenas, 77.3 y $49.1 \%$ de los autoadscritos y los hablantes, respectivamente, se encuentran en escuelas regulares. Asimismo, 59.9 y $38.9 \%$ de ambos grupos viven en localidades mayores a 2,500 habitantes. La mayoría de estos niños vive en localidades de alta o muy alta marginación, a diferencia de lo que ocurre con los niños no indígenas.

\footnotetext{
${ }^{7}$ Por razones de simplicidad, en adelante este grupo se denomina "hablantes".
} 
Tabla I. Estadísticos descriptivos de las variables de interés por condición étnico-lingüística de los alumnos

\begin{tabular}{|c|c|c|c|c|c|c|}
\hline & \multicolumn{2}{|c|}{ Identidad } & \multicolumn{2}{|c|}{ Lengua } & \multirow{2}{*}{ Total } & \multirow{2}{*}{ Válidos } \\
\hline & No & Sí & No & Sí & & \\
\hline \multicolumn{7}{|l|}{ Características de las escuelas } \\
\hline Tipo de servicio & & & & & & 100.0 \\
\hline General pública & 88.8 & 77.3 & 88.2 & 49.1 & 87.0 & \\
\hline Indígena & 1.4 & 17.9 & 2.5 & 48.9 & 3.9 & \\
\hline Privada & 9.9 & 4.8 & 9.3 & 2.0 & 9.1 & \\
\hline \multicolumn{6}{|l|}{ Tamaño de localidad (habitantes) } & 100.0 \\
\hline No identificada & 0.1 & 0.1 & 0.1 & 0.1 & 0.1 & \\
\hline 1 a 499 & 6.8 & 15.1 & 7.7 & 20.2 & 8.0 & \\
\hline 500 a 2499 & 13.1 & 25.1 & 14.2 & 40.9 & 14.9 & \\
\hline 2500 a 99999 & 30.7 & 32.6 & 31.3 & 20.9 & 31.0 & \\
\hline 100000 o más & 49.4 & 27.2 & 46.8 & 18.0 & 46.0 & \\
\hline \multicolumn{6}{|l|}{ Índice de marginación de la localidad } & 100.0 \\
\hline No identificada & 0.6 & 0.6 & 0.6 & 1.4 & 0.6 & \\
\hline Muy alta / Alta & 33.6 & 61.3 & 36.7 & 76.4 & 37.9 & \\
\hline Media & 32.0 & 22.1 & 31.0 & 13.1 & 30.4 & \\
\hline Muy baja / Baja & 33.9 & 16.0 & 31.8 & 9.1 & 31.1 & \\
\hline Multigrado & 7.2 & 13.5 & 8.0 & 14.8 & 8.2 & 100.0 \\
\hline $\begin{array}{l}\text { Todos los maestros esperan que los } \\
\text { alumnos alcancen la universidad }\end{array}$ & 44.9 & 33.9 & 43.7 & 25.3 & 43.2 & 100.0 \\
\hline Director con grupo a cargo & 13.7 & 23.3 & 14.7 & 29.9 & 15.2 & 100.0 \\
\hline \multicolumn{7}{|l|}{ Características del entorno escolar } \\
\hline $\begin{array}{l}\text { Índice de bienes en el hogar } \\
\text { (promedio escolar) }\end{array}$ & 0.1 & -0.8 & 0.1 & -1.8 & 0.0 & \\
\hline $\begin{array}{l}\text { Porcentaje de alumnos que se } \\
\text { identifican como indígenas en la escuela }\end{array}$ & 11.8 & 34.9 & 14.3 & 51.7 & 15.4 & 100.0 \\
\hline $\begin{array}{l}\text { Porcentaje de alumnos hablantes de } \\
\text { Lengua indígena en la escuela }\end{array}$ & 1.7 & 9.9 & 1.8 & 39.8 & 2.9 & 100.0 \\
\hline \multicolumn{7}{|l|}{ Características alumno-escuela } \\
\hline Alumno recibe clases en lengua indígena & 5.5 & 22.6 & 6.9 & 48.9 & 8.1 & 100.0 \\
\hline Percepción del clima de aula & 0 & 0.00 & 0.01 & -0.33 & 0.00 & 99.4 \\
\hline \multicolumn{7}{|l|}{ Características de los alumnos } \\
\hline Índice de bienes en el hogar & 0.0 & -0.7 & -0.1 & -1.4 & 0.0 & 100.0 \\
\hline Nivel educativo máximo del hogar & & & & & & 84.9 \\
\hline Hasta primaria & 13.1 & 31.2 & 12.7 & 37.7 & 13.4 & \\
\hline Secundaria o Bachillerato & 54.0 & 45.8 & 53.5 & 46.4 & 53.3 & \\
\hline Universidad o más & 32.9 & 15.0 & 33.8 & 16.0 & 33.4 & \\
\hline \multicolumn{6}{|l|}{ Asistencia a preescolar (años) } & 99.7 \\
\hline 0 & 18.5 & 18.4 & 18.4 & 23.7 & 18.5 & \\
\hline 1 & 7.2 & 8.7 & 7.4 & 7.9 & 7.4 & \\
\hline 2 & 24.9 & 19.8 & 24.4 & 15.5 & 24.1 & \\
\hline 3 & 49.4 & 53.2 & 49.9 & 53.0 & 50.0 & \\
\hline \multicolumn{6}{|l|}{ Reprobó algún grado } & 99.8 \\
\hline No & 87.4 & 80.7 & 86.8 & 73.4 & 86.4 & \\
\hline Sí & 12.6 & 19.3 & 13.2 & 26.7 & 13.6 & \\
\hline \multicolumn{6}{|l|}{ Expectativas educativas } & 99.8 \\
\hline Hasta bachillerato & 23.1 & 31.8 & 23.7 & 48.4 & 24.5 & \\
\hline Hasta licenciatura & 30.2 & 28.5 & 30.1 & 26.7 & 30.0 & \\
\hline Hasta posgrado & 46.6 & 39.8 & 46.2 & 24.9 & 45.6 & \\
\hline \multicolumn{6}{|l|}{ Alumno trabaja } & 99.7 \\
\hline No & 63.7 & 48.9 & 62.3 & 33.4 & 61.4 & \\
\hline Sí, en su hogar & 26.5 & 37.3 & 27.6 & 47.2 & 28.1 & \\
\hline Sí, fuera de su hogar & 9.8 & 13.8 & 10.2 & 19.4 & 10.5 & \\
\hline $\begin{array}{l}\text { Promedio de aprendizajes (lenguaje y } \\
\text { comunicación) }\end{array}$ & 507.8 & 476.9 & 505.3 & 430.7 & 503.1 & 100.0 \\
\hline Promedio de aprendizajes (matemáticas) & 506.2 & 482.1 & 504.3 & 443.6 & 502.5 & 100.0 \\
\hline
\end{tabular}

Fuente: elaboración propia con base en microdatos de Planea 2015. Muestra restringida para los análisis. 
Los alumnos indígenas tienden a provenir de hogares con menores niveles de bienestar y educación; tienen mayores índices de reprobación, menores expectativas educativas y mayor participación en situaciones de trabajo infantil. También tienen una propensión mayor a asistir a escuelas multigrado, escuelas donde los maestros tienen menores expectativas educativas hacia los alumnos, donde el director tiene un grupo a cargo, y con mayores carencias de materiales educativos. Asimismo, tienden a asistir a escuelas con niveles inferiores de composición socioeconómica. Particularmente, es la población hablante la que muestra las mayores desventajas en estos indicadores.

En las dos últimas filas de la tabla I se muestran los promedios de aprendizaje para las poblaciones de interés, expresados en los puntajes originales. Como puede verse, las brechas van de 61 a 75 puntos en desventaja de los hablantes, y de 24 a 31 puntos en desventaja de los autoadscritos, respectivamente para matemáticas y lengua. Se trata de diferencias muy importantes, sobre todo en el caso de los hablantes. ¿En qué medida las condiciones sociales y escolares expuestas logran agotar la explicación de las brechas de aprendizaje? ¿Qué puede decirse de las brechas remanentes, en relación con las otras explicaciones disponibles? A continuación, se presentan los resultados de los análisis.

\subsection{Modelos jerárquico-lineales}

La tabla II presenta los resultados de los modelos para lenguaje/comunicación y matemáticas. El objetivo principal de este análisis es mostrar los puntos de partida de las brechas indígenas, su reducción a medida que se introducen diferentes variables, y la posible existencia de interacciones entre ellas. Por razones de espacio y simplicidad en la presentación, sólo se muestran los coeficientes relevantes para este objetivo. ${ }^{8}$

\footnotetext{
${ }^{8}$ En particular, se omite el conjunto de variables socioeconómicas y escolares de los modelos 3 y 4.
} 
Tabla II. Coeficientes de regresión sobre resultados de aprendizaje para variables relacionadas

con la condición étnica del alumno y la composición étnica de la escuela, por área evaluada

\begin{tabular}{|c|c|c|c|c|c|c|c|c|c|c|}
\hline & \multicolumn{5}{|c|}{ Lenguaje y comunicación } & \multicolumn{5}{|c|}{ Matemáticas } \\
\hline & 1 & 2 & 3 & 4 & 5 & 1 & 2 & 3 & 4 & 5 \\
\hline $\begin{array}{l}\text { Alumno se identifica } \\
\text { indígena }\end{array}$ & $\begin{array}{l}-0.111 \\
(-7.41)^{* * *}\end{array}$ & $\begin{array}{l}-0.095 \\
(-6.17)^{* * *}\end{array}$ & $\begin{array}{l}-0.065 \\
(-4.78)^{* * *}\end{array}$ & $\begin{array}{l}-0.080 \\
(-6.06)^{* * *}\end{array}$ & $\begin{array}{l}-0.159 \\
(-7.75)^{* * *}\end{array}$ & $\begin{array}{l}-0.101 \\
(-7.00)^{* * *}\end{array}$ & $\begin{array}{l}-0.088 \\
(-5.99)^{* * *}\end{array}$ & $\begin{array}{l}-0.060 \\
(-4.12)^{* * *}\end{array}$ & $\begin{array}{l}-0.074 \\
(5.24) * * *\end{array}$ & $\begin{array}{l}-0.143 \\
(-6.49 * * *)\end{array}$ \\
\hline $\begin{array}{l}\text { Alumno habla lengua } \\
\text { indígena }\end{array}$ & $\begin{array}{l}-0.252 \\
(-5.96) * * *\end{array}$ & $\begin{array}{l}-0.173 \\
(-4.03)^{* * *}\end{array}$ & $\begin{array}{l}-0.092 \\
(-2.37)^{*}\end{array}$ & $\begin{array}{l}-0.078 \\
(-2.12) *\end{array}$ & $\begin{array}{l}-0.145 \\
(-2.94)^{* *}\end{array}$ & $\begin{array}{l}-0.221 \\
(-6.61) * * *\end{array}$ & $\begin{array}{l}-0.148 \\
(-4.31)^{* * *}\end{array}$ & $\begin{array}{l}-0.080 \\
(-2.47)^{*}\end{array}$ & $\begin{array}{l}-0.068 \\
(-2.15) *\end{array}$ & $\begin{array}{l}-0.144 \\
(-3.36)^{* *}\end{array}$ \\
\hline $\begin{array}{l}\text { Porcentaje de alumnos que } \\
\text { se identifican como } \\
\text { indígenas en la escuela }\end{array}$ & & $\begin{array}{l}-0.003 \\
(-2.21)^{*}\end{array}$ & $\begin{array}{l}0.007 \\
(6.15)^{* * *}\end{array}$ & $\begin{array}{l}0.003 \\
(3.61)^{* * *}\end{array}$ & $\begin{array}{l}0.002 \\
(2.32)^{*}\end{array}$ & & $\begin{array}{l}-0.001 \\
(-0.84)\end{array}$ & $\begin{array}{l}0.006 \\
(5.06)^{* * *}\end{array}$ & $\begin{array}{l}0.003 \\
(2.34)^{*}\end{array}$ & $\begin{array}{l}0.002 \\
(1.30)\end{array}$ \\
\hline $\begin{array}{l}\text { Porcentaje de alumnos que } \\
\text { hablan lengua indígena en } \\
\text { la escuela }\end{array}$ & & $\begin{array}{l}-0.009 \\
(-6.14) * * *\end{array}$ & $\begin{array}{l}-0.006 \\
(-3.81) * * *\end{array}$ & $\begin{array}{l}-0.005 \\
(-3.60) * * *\end{array}$ & $\begin{array}{l}-0.007 \\
(-4.40) * * *\end{array}$ & & $\begin{array}{l}-0.009 \\
(-5.86)^{* * *}\end{array}$ & $\begin{array}{l}-0.006 \\
(-3.85)^{* * *}\end{array}$ & $\begin{array}{l}-0.005 \\
(-3.40) * *\end{array}$ & $\begin{array}{l}-0.007 \\
(-4.31)^{* * *}\end{array}$ \\
\hline Identifica*\%Identifica & & & & & $\begin{array}{l}0.003 \\
(5.44)^{* * *}\end{array}$ & & & & & $\begin{array}{l}0.003 \\
\left(4.26^{* * *}\right)\end{array}$ \\
\hline Habla*\%Habla & & & & & $\begin{array}{l}0.003 \\
(1.88) \\
\end{array}$ & & & & & $\begin{array}{l}0.004 \\
(2.87)^{* *}\end{array}$ \\
\hline ICC & & & & & 0.330 & & & & & 0.285 \\
\hline Seudo $\mathrm{R}^{2}$ Nivel 2 & & & & & 0.763 & & & & & 0.598 \\
\hline $\begin{array}{l}\text { Seudo } \mathrm{R}^{2} \text { Nivel } 1 \\
\mathrm{R}^{2} \text { global }\end{array}$ & & & & & $\begin{array}{l}0.160 \\
0.359\end{array}$ & & & & & $\begin{array}{l}0.112 \\
0.250\end{array}$ \\
\hline
\end{tabular}

Fuente: Elaboración propia con base en microdatos de Planea 2015, 60. grado de Primaria. Los Modelos 3 y 4 contienen, respectivamente, controles por condiciones del alumno y [condiciones del alumno + condiciones de la escuela] (se omiten coeficientes por razones de espacio y claridad).

Errores estándar entre paréntesis. ***: $p<0.001 ; * *: p<0.01 ; *: p<0.05$ 
El modelo 1 incluye los efectos de autoadscripción y habla indígena (variables 1 y 2). ${ }^{9}$ El modelo 2 agrega el porcentaje de alumnos autoadscritos y hablantes en la escuela (variables 15 y 16). El 3 incluye variables socioeconómicas, de trayectoria escolar y las expectativas educativas del alumno, así como el promedio escolar del bienestar económico (variables 3 a 9 y 17). El 4 agrega las variables referentes a la escuela: modalidad, recursos, clima y expectativas de los maestros (variables $10,11,14,18$ y 19). ${ }^{10}$ En el modelo 5 se agregan las interacciones entre la condición étnica del alumno y el porcentaje de indígenas en su escuela, con los dos criterios ( $1 \times 15$ y $2 \times 16)$.

Si se atiende a los coeficientes de la tabla II, el modelo 1 muestra que tanto los que hablan una lengua indígena como aquellos que se identifican como indígenas tienen resultados inferiores en ambas áreas, y la desventaja es mayor para los primeros. No se observan diferencias significativas en el tamaño de las brechas entre las áreas evaluadas.

El modelo 2 muestra que el porcentaje de alumnos que hablan una lengua indígena en la escuela tiene efectos significativos y separados de los efectos individuales. Esto es relevante porque indica que una parte de la brecha no se genera entre individuos sino entre escuelas, en función de cómo está compuesto el alumnado. Se trata, en principio, de efectos de magnitud considerable: la desventaja asociada a asistir a una escuela con $32.2 \%$ de indígenas hablantes (promedio de las escuelas indígenas) vs una escuela con $1.5 \%$ de indígenas hablantes (promedio de las escuelas generales) es de 0.28 desvíos estándar. En cambio, el porcentaje de alumnos que se identifican como indígenas no arroja efectos significativos en matemáticas, y sólo tiene efectos débiles en lenguaje.

Cuando se controlan los coeficientes por las características sociales y educativas de los alumnos, así como por la composición socioeconómica de las escuelas (modelo 3), se observan cambios importantes. En primer lugar, en ambas áreas de aprendizaje se reducen los efectos negativos de la autoadscripción y el habla (particularmente estos últimos). También se reducen los coeficientes asociados al porcentaje escolar de alumnos que hablan una lengua indígena, lo que indica que una parte importante de esta brecha podría explicarse por las diferencias en la composición socioeconómica de estos grupos. Por último, el porcentaje de quienes se identifican como indígenas en la escuela toma un signo positivo, lo que podría indicar la presencia de dinámicas culturales colectivas, asociadas a la defensa de una identidad indígena, con efectos positivos sobre los aprendizajes. ${ }^{11}$

En el modelo 4 (variables escolares), el cambio corresponde, precisamente, al coeficiente asociado al porcentaje de alumnos que se identifican como indígenas, que se reduce en comparación con el modelo 3. Esto sugiere que parte de la explicación de este efecto residiría en que el porcentaje de autoadscripción indígena en la escuela se asocia a un mejor clima de aula y a expectativas educativas de los maestros más elevadas. ${ }^{12}$

Finalmente, el modelo 5 muestra una interacción significativa, positiva en ambas áreas, entre el porcentaje de quienes se identifican como indígenas en la escuela y la autoidentificación individual. En el caso de la interacción para quienes hablan una lengua indígena, sólo es significativa para matemáticas,

\footnotetext{
${ }^{9}$ Se tomó la decisión de introducir las variables por separado (y no su combinación lógica como dummies) por las siguientes razones: 1) Quienes declaran haber aprendido primero una lengua indígena (2.4\% del total) no son un subconjunto de los que se identifican como tales: $49.9 \%$ de los hablantes no se identifican como indígenas. 2) Este grupo representa un porcentaje muy bajo del total de evaluados (1.2\%), por lo que se dificulta analizarlos por separado. 3) $90.7 \%$ del 13.1 que se identifica como indígena no aprendió a hablar primero una lengua indígena.

${ }^{10}$ Como parte de los análisis previos, se especificó un modelo alternativo con dos variables escolares de tipo institucional: escuela multigrado y director con grupo a cargo (\#12 y \#13), ninguna de las cuales resultó significativa.

${ }^{11}$ Todos los coeficientes no presentados en el modelo 3 muestran la relación esperada con los aprendizajes: el nivel educativo de los padres, el nivel socioeconómico del hogar (a nivel individual y agregado), la asistencia al preescolar y las expectativas educativas se asocian con una mejora en los aprendizajes. Por su parte, haber reprobado, y estar trabajando (especialmente fuera del hogar) se asocian con un decremento en los aprendizajes.

${ }^{12}$ Tres elementos son especialmente destacables de los coeficientes no presentados en el modelo 4: 1) una vez controladas las variables socioeconómicas y escolares, las escuelas indígenas no muestran un efecto significativo; 2) las escuelas privadas, en cambio, mantienen un fuerte efecto de signo positivo; 3) finalmente, la percepción individual del clima de aula tiene efectos positivos pronunciados (alrededor de 0.14 desvíos estándar en cada una de las áreas evaluadas, por cada unidad de incremento del índice).
} 
también con signo positivo. Estos resultados apuntan a que, una vez descontados los efectos del origen social, las escuelas con mayor proporción de indígenas tienden a ser más favorables para el aprendizaje de los alumnos indígenas, lo cual podría apuntar a la existencia de fenómenos de discriminación cultural que perjudican a los alumnos indígenas que se encuentran en minoría.

Un ejercicio realizado a partir de los puntajes predichos por el modelo 5 mostró que las diferencias favorables a los no indígenas en las escuelas generales tienden a desaparecer en las escuelas con alta presencia de indígenas autoadscritos, a través de la mejora de los aprendizajes de ambos tipos de alumnos, pero fundamentalmente de quienes se identifican como indígenas (manteniendo el resto de los factores constantes). ${ }^{13}$ El resultado es el inverso cuando se realiza el ejercicio para escuelas con alta proporción de alumnos hablantes: los aprendizajes de indígenas y no indígenas tienden a descender para todos, y sobre todo para los no indígenas, hasta hacerse indistinguibles estadísticamente.

\subsection{Descomposiciones Oaxaca-Blinder}

Las descomposiciones O-B se realizaron como contrastes separados entre no indígenas vs autoadscritos y no indígenas vs hablantes, a partir de modelos que incorporan las mismas variables de los jerárquicolineales ${ }^{14}$ (ver tabla III).

Tabla III. Brechas de aprendizaje entre no indígenas, indígenas por identificación e indígenas por lengua, explicadas por los modelos lineales y descompuestas en componentes explicativos*

\begin{tabular}{|c|c|c|c|c|c|c|c|c|}
\hline & \multicolumn{4}{|c|}{ Lenguaje y comunicación } & \multicolumn{4}{|c|}{ Matemáticas } \\
\hline Puntajes & Identidad & $\%$ & Lengua & $\%$ & Identidad & $\%$ & Lengua & $\%$ \\
\hline No indígena & 0.006 & & -0.020 & & 0.005 & & -0.013 & \\
\hline Indígena & -0.297 & & -0.762 & & -0.224 & & -0.603 & \\
\hline Brecha & 0.303 & & 0.742 & & 0.229 & & 0.590 & \\
\hline Explicada & 0.256 & 0.846 & 0.640 & 0.862 & 0.189 & 0.825 & 0.514 & 0.872 \\
\hline No explicada & 0.047 & & 0.103 & & 0.040 & & 0.076 & \\
\hline \multicolumn{9}{|l|}{ Explicado por... } \\
\hline Origen social & 0.056 & 0.219 & 0.104 & 0.163 & 0.034 & 0.181 & 0.057 & 0.110 \\
\hline Entorno escolar & 0.117 & 0.455 & 0.279 & 0.436 & 0.066 & 0.352 & 0.185 & 0.361 \\
\hline Trayectoria escolar & 0.062 & 0.240 & 0.162 & 0.253 & 0.061 & 0.321 & 0.157 & 0.305 \\
\hline Escuela & 0.022 & 0.085 & 0.095 & 0.148 & 0.028 & 0.146 & 0.115 & 0.224 \\
\hline
\end{tabular}

Fuente: Elaboración propia con base en microdatos de Planea 2015, 60. grado de primaria.

Todas las cifras son significativamente distintas de 0 , con $p<0.05$, a excepción de aquellas en cursiva. Alumnos: 81,$389 ;$ Escuelas: 2,952. Referencia: Origen=sexo; índice de bienestar en el hogar; máximo nivel educativo del hogar; alumno trabaja; Entorno=índice de bienestar promedio de la escuela; porcentaje de hablantes de lengua indígena en la escuela; Trayectoria=alumno cursó preescolar; alumno reprobó primaria; expectativas educativas del alumno; Escuela=modalidad; clima de aula percibido por alumnos. * Expresadas en unidades de desvío estándar (normal) y como porcentajes (negritas).

La proporción total de la brecha explicada por los modelos (filas 3 a 5) es similar en ambas áreas de aprendizaje, y similar también para las comparaciones con indígenas por identidad e indígenas hablantes; de manera general, el porcentaje explicado se mueve en un rango elevado, de 83 a $87 \%$, lo que da cuenta de un buen rendimiento explicativo de los modelos. Al igual que en el análisis jerárquico de la sección anterior, el hecho de que no se observe una proporción "extra" no explicada en lenguaje, o una proporción "extra" no explicada para los hablantes, debilita la plausibilidad de la hipótesis relacionada con el idioma de la prueba como factor explicativo de las desventajas de aprendizaje.

El análisis de los componentes explicados de cada brecha (filas 6 a 9) revela un patrón interesante. En términos generales, es el entorno escolar el que tiene un mayor peso explicativo, seguido de la

\footnotetext{
${ }^{13}$ Realicé simulaciones de resultados en ambas pruebas comparando escuelas con $12 \%$ y $65 \%$ de alumnos que se identifican como indígenas (porcentajes promedio para las escuelas generales e indígenas, respectivamente).

${ }^{14}$ La excepción es que, por razones de parsimonia, y dado que el modelo tiene por objetivo explicar la brecha de aprendizajes inicial, en lugar de identificar posibles efectos de variables se omitió el porcentaje de indígenas por autoadscripción de las escuelas, así como las interacciones entre variables individuales y escolares.
} 
trayectoria escolar del alumno. La escuela y el origen social individual, por su parte, tienen pesos menores. No obstante, el peso explicativo de los factores analizados varía sistemáticamente dependiendo del área de conocimiento y del tipo de contraste evaluado. Los factores de la estructura social (origen social del alumno y entorno social escolar) tienen más peso en la prueba de lenguaje que en la de matemáticas, y más peso en la brecha de los indígenas por autoadscripción que en los indígenas por lengua. De éstos, el entorno escolar (que reúne nivel socioeconómico agregado y porcentaje de indígenas) muestra de forma sistemática un peso entre dos y tres veces mayor que los factores socioeconómicos individuales.

Adicionalmente, la trayectoria escolar del alumno (preescolar, reprobación y expectativas educativas) tiene un mayor peso explicativo en matemáticas que en lenguaje. Los factores escolares, por su parte, también incrementan su peso en matemáticas, y en el contraste con indígenas hablantes. Las implicaciones de estos resultados se discuten a continuación.

\section{Discusión}

Si bien en México no se cuenta con datos que permitan evaluar directamente el rendimiento de todas las explicaciones posibles para la desventaja de aprendizajes de los indígenas, el análisis de las pruebas de aprendizaje Planea y sus cuestionarios de contexto arrojan algunos elementos de prueba indirectos, así como hallazgos novedosos.

Los modelos presentados lograron explicar una parte importante de las brechas, tanto por autoadscripción como por lengua. La parte "no explicada", que suele interpretarse como el efecto de la discriminación (en este caso, "discriminación + irrelevancia del modelo educativo"), ronda el 15\%. Sin embargo, como se expone a continuación, no es sencillo separar los efectos de los recursos de los eventuales mecanismos de discriminación cultural.

Se destaca el efecto de la composición étnico-lingüística de las escuelas, distinto al de la composición socioeconómica y distinto al de las características individuales, que sugiere que una parte importante de las brechas podría obedecer a las características culturales de las interacciones escolares. De manera sorprendente, una vez que se controlan los factores socioeconómicos, un mayor porcentaje de indígenas por autoadscripción en la escuela mejora los aprendizajes de todos los alumnos, una vez controladas las características socioeconómicas. Este resultado parece contra-intuitivo, pero debe recordarse que emerge de mantener "constantes" las variables socioeconómicas, un ejercicio cuya validez no está exenta de críticas. Una posible explicación de este fenómeno es que altos porcentajes de autoadscripción corresponden a comunidades con mayores niveles de cohesión social, los cuales, a su vez, pueden favorecer procesos de enseñanza-aprendizaje adecuados a las necesidades de los alumnos.

También se destaca el efecto negativo que tiene la composición lingüística de la escuela, incluso después de controlar por factores socioeconómicos. Debe recordarse que esta composición afecta de manera significativa a pocas escuelas (sólo $4 \%$ de las escuelas tiene un porcentaje igual o superior a $10 \%$ de alumnos hablantes), por lo que se trata de un fenómeno localizado. En este sentido, estos efectos podrían estar ocultando los de otras características no observadas, como prácticas de enseñanza inadecuadas, menor calidad de los docentes, o dificultades para avanzar en la implementación del currículum.

Si bien los factores socioeconómicos individuales logran explicar una parte importante de las brechas, abonando a la hipótesis de que las desigualdades socioeconómicas superan a los eventuales efectos de la discriminación cultural, también es cierto que no son los únicos involucrados en esta explicación. Por sí solos (sumando los factores individuales y el bienestar agregado a nivel escolar) explican entre 30 y $60 \%$ del total de las brechas, con mayor efecto en lenguaje y en el contraste por autoadscripción. La trayectoria escolar del propio alumno, por otra parte, explica entre 24 y 32\% de las diferencias.

En este punto es importante destacar que la trayectoria "individual" incluye eventos como la reprobación y la formación de expectativas educativas, sobre los cuales no puede descartarse la influencia de mecanismos de discriminación o irrelevancia cultural. En este sentido, las trayectorias nunca son 
"individuales" porque están condicionadas por un sistema educativo que puede operar contra los alumnos provenientes de minorías culturales.

Otro hallazgo relevante es que en casi todos los contrastes las escuelas tienen una incidencia significativa, con mayor peso en el contraste con los hablantes para la prueba de matemáticas. Esta incidencia está vinculada principalmente al sector (público vs privado) y a la percepción del clima de aula por parte de los alumnos. De nuevo, en este último caso no puede descartarse la incidencia de actitudes discriminatorias que afecten las relaciones entre maestros y alumnos.

Por último, es interesante notar que el origen socioeconómico y el entorno escolar tienen un mayor peso en las brechas de lenguaje que en las de matemáticas, y tienden a ser mayores también en la explicación de los contrastes por autoadscripción que en los contrastes por lengua. Lo primero coincide con lo observado en otras investigaciones a nivel regional, y se explicaría porque el aprendizaje de las matemáticas es más dependiente de los procesos escolares. Lo segundo parecería indicar que -al menos en lo que concierne a los aprendizajes escolares evaluados- los alumnos hablantes son más dependientes de las escuelas para alcanzarlos, dado que es el único recurso al que pueden acceder. Esto destaca la necesidad de mejorar sustancialmente la calidad de la oferta escolar que reciben estos alumnos.

En síntesis, si bien el análisis permite afirmar que una buena parte de las brechas entre indígenas y no indígenas obedecen a diferencias de recursos socioeconómicos, y no a procesos de discriminación o a la irrelevancia cultural del modelo educativo, las posibles interacciones entre los factores involucrados en la brecha de aprendizajes desdibujan la frontera entre lo que puede atribuirse a diferencias en los recursos y diferencias atribuibles a procesos de discriminación.

La reprobación y la formación de expectativas educativas, en particular, son aspectos de la trayectoria escolar a los que debería atenderse más de cerca, dado que pueden ser directamente influidos por prácticas de discriminación o marginación cultural. Asimismo, el hecho de que los alumnos indígenas tiendan a tener mejores resultados en escuelas con mayor proporción de alumnos similares (una vez controlados los factores socioeconómicos) sugiere que la dispersión de las minorías culturales acarrea efectos negativos en los aprendizajes de sus miembros, lo que podría apuntar a la existencia de procesos de marginación cultural o de debilitamiento del capital social basado en lazos comunitarios.

Por otra parte, a nivel de las escuelas, es claro que factores no observados podrían estar incidiendo de manera significativa y distorsionando las asociaciones con las variables observadas, por lo que en el futuro es necesario desarrollar instrumentos que permitan visibilizarlos. Sobre todo, sería necesario enfocarse en la habilidad y la capacidad pedagógica de los docentes (uno de los pendientes más importantes en cuanto a instrumentos de observación, y también en cuanto a la política educativa mexicana).

\section{Referencias}

Backhoff, E., Solano, G., Contreras, L., Vázquez, M. y Sánchez, A. (2015). ¿Son adecuadas las traducciones para evaluar los aprendizajes de los estudiantes indígenas? Un estudio con preescolares mayas. México: Instituto Nacional para la Evaluación de la Educación.

Barriga, R. (2008). Miradas a la interculturalidad: el caso de una escuela urbana con niños indígenas. Revista Mexicana de Investigación Educativa, 13(39), 1229-1254. Recuperado de https://www.comie.org.mx/revista/v2018/rmie/index.php/nrmie/article/view/597

Bastiani, J., Ruiz-Montoya, L., Estrada, E., Cruz, T. y Aparicio, J. (2012). Política educativa indígena. Práctica docente, castellanización, burocracia y centralización de la educación como limitaciones del éxito pedagógico en la región Ch'ol, Chiapas. Perfiles Educativos, 34(135), 8-25. Recuperado de http://www.iisue.unam.mx/perfiles/index.php?numero=135\&anio=2012

Bertely, M. (2002). Panorama histórico de la educación para los indígenas en México. Diccionario de la historia de la educación en México. México: CONACYT/CIESAS. 
Blinder, A. S. (1973). Wage discrimination: reduced form and structural estimates. Journal of Human Resources, 8(4), 436-455. doi:10.2307/144855

Cervini, R. (2012). El "efecto escuela" en países de América Latina: reanalizando los datos del SERCE. Archivos Analíticos de Políticas Educativas, 20(39), 1-27. doi:10.14507/epaa.v20n39.2012

Consejo Nacional de Evaluación de la Política de Desarrollo Social. (2014). La pobreza en la población indigena de México, 2012. México: Autor.

Fernández, T. (2007). Distribución del conocimiento escolar: clases sociales, escuelas y sistema educativo en América Latina. México: El Colegio de México.

Hernández-Zavala, M., Patrinos, H., Sakellariou, Ch. y Shapiro, J. (2006). Quality of schooling and quality of schools for indigenous students in Guatemala, Mexico, and Peru. Documento de trabajo Núm. 3982 sobre investigación de políticas del Banco Mundial.

Instituto Nacional de Estadística, Geografía e Informática. (2015). Panorama Sociodemográfico de México. 2015. México: Autor.

Instituto Nacional para la Evaluación de la Educación. (2015). Resultados de la consulta previa, libre e informada a pueblos y comunidades indígenas sobre la evaluación educativa. México: Autor.

Instituto Nacional para la Evaluación de la Educación. (2016a). Panorama Educativo de México, 2015. Indicadores del Sistema Educativo Nacional. México: Autor.

Instituto Nacional para la Evaluación de la Educación. (2016b). Infraestructura, mobiliario y materiales de apoyo educativo en las escuelas primarias. ECEA 2014. México: Autor.

McEwan, P. (2004). The indigenous test score gap in Bolivia and Chile. Economic Development and Cultural Change, 53(1), 157-190. doi:10.1086/423257

McEwan, P. y Trowbridge, M. (2007). The achievement of indigenous students in Guatemalan primary schools. International Journal of Education Development, 27(1), 61-76.

Mijangos-Noh, J. (2009). Racism against the Mayan Population in Yucatan, Mexico: How Current Education Contradicts the Law. Ponencia presentada en el Encuentro Anual de la American Educational Research Association, San Diego, California. Recuperado de https://files.eric.ed.gov/fulltext/ED505698.pdf

Oaxaca, R. (1973). Male-female wage differentials in urban labor markets. International Economic Review, 14(3), 693-709.

Raesfeld, L. (2009). Niños indígenas en escuelas multiculturales. Trayectorias, 11(28), 38-57.

Raudenbush, S. W. y Bryk, A. S. (2002). Hierarchical linear models: applications and data analysis methods (Vol. 1). EUA: University of Chicago.

Santibáñez, L. (2016). The indigenous achievement gap in Mexico: The role of teacher policy under intercultural bilingual education. International Journal of Educational Development, 47, 63-75.

Sakellariou, Ch. (2008). Peer effects and the indigenous/non-indigenous early test-score gap in Peru. Education Economics, 16(4), 371-390.

Schmelkes, S. (2013). Educación para un México intercultural. Sinéctica, 40, 1-12. 
Tinajero, G. y Englander, K. (2011). Bilingual-intercultural education for indigenous children: the case of Mexico in an era of globalizationand uprisings, Intercultural Education, 22(3), 163-178.

doi:10.1080/14675986.2011.592019

Treviño, E. (2013). Learning inequality among indigenous students in Mexico, en B. Jensen y A. Sawyer (Eds.), Regarding education: mexican-american schooling, immigration, and bi-national improvement (pp. 95123. Nueva York: Teachers College.

Villarreal A. (2014). Ethnic identification and its consequences for measuring inequality in Mexico. American Sociological Review, 79(4), 775-806. 